sen psychosoziale Risikofaktoren der Familie und die Reaktionen der Eltern auf die Bedürfnisse des Kindes erfasst werden. Bei der körperlichen Untersuchung des Säuglings müssen u. a. Hydratation und subkutanes Fett kontrolliert werden. Zudem muss auf eventuelle Abnormitäten einschließlich Zungenanomalien hin untersucht werden.

Unter den Infektionen sind es vor allem Harnwegsinfekte, die für Unmut bei den Kleinen sorgen. Dagegen können Babys, die auf Berührung, Bewegung oder Geräusche mit Schreien reagieren, an einer sensorischen Übererregbarkeit leiden. Warnhinweise für eine Entwicklungsstörung sind v. a. ein seltener Augenkontakt sowie mangelndes Interesse an Interaktionen mit den Eltern.

\section{Wie verkraften es die Eltern?}

Eine Befragung von über 3000 Familien hat ergeben, dass 6\% der Eltern ihr Baby, wenn es schrie, körperlich in unangemessener Weise behandelten. Eltern, die in Anbetracht des Gebrülls selbst in Rage geraten, sollten das Kind an einem sicheren Ort ablegen, sich entfernen und erst zurückkehren, wenn sie sich wieder unter Kontrolle haben. Studien haben gezeigt, dass dieses Vorgehen die Zahl der körperlichen Übergriffe auf das Kind reduzieren kann.

Viele Eltern fühlen sich auch schuldig, wenn ihr Kind immer wieder ohne ersichtlichen Grund schreit. So können Depressionen bei der Mutter entstehen. Allerdings ist es umgekehrt auch möglich, dass ein Kind durch das Schreien die Zuwendung einfordert, die ihm eine depressive Mutter nicht ausreichend geben kann.

ST =

- Quelle: Douglas P., Hill P. Managing infants who cry excessively in the first few months of life. BMJ 2011;343:1265-1269

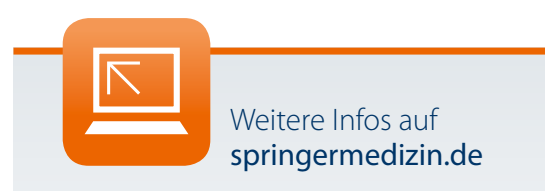

Stillen \& Co. online unter www.springermedizin.de/ stillen

Nicht nur akut bedrohlich

\title{
Venenthrombosen erhöhen die Mortalität für Jahre
}

\begin{abstract}
Die schlechte Prognose nach einer venösen Thromboembolie beschränkt sich nicht auf die ersten Monate nach dem Ereignis. Das Sterberisiko ist bis zu acht Jahre lang erhöht, wie eine Fall-Kontroll-Studie der Universität von Leiden jetzt enthüllt.
\end{abstract}

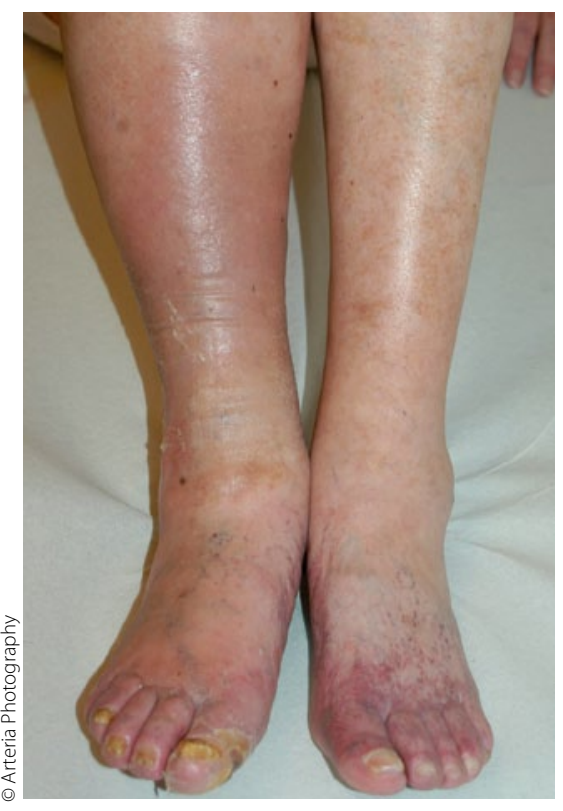

Auch wenn kein Tumor dahinter steckt, ist die Sterblichkeit nach einer Thrombose langfristig erhöht.

— In der Studie wurden 4947 Patienten, die eine erste, nicht tödliche Venenthrombose oder Lungenembolie erlitten hatten, 6154 Kontrollpersonen ohne Thrombose im Alter von 18 bis 70 Jahren gegenübergestellt. Die Patienten wurden bis zu acht Jahre lang verfolgt, im Median 5,5 Jahre. Todesfälle und -ursachen wurden anhand des niederländischen Sterberegisters ermittelt.

Im Beobachtungszeitraum starben 601 Thrombosepatienten und 135 Kontrollpersonen. Mit 22,7 im Vergleich zu 4,7 Todesfällen pro 1000 Personenjahren war die Sterblichkeit in der Thrombosegruppe vierfach erhöht. Die höchste Mortalität hatten erwartungsgemäß Thrombosepatienten mit einer malignen
Erkrankung: Von ihnen starben $55 \%$ bis zum Studienende, damit war ihre Mortalität 17-mal so hoch wie in der Kontrollgruppe. Selbst im Vergleich zu Krebspatienten ohne Thrombose hatten sie noch eine fünffach höhere Sterberate.

\section{Auch ohne Malignom sinkt die Lebenserwartung}

Bei Thrombosepatienten ohne Malignom war die Sterblichkeit doppelt so hoch wie in der Kontrollgruppe. Das höchste Risiko bestand in den ersten drei Jahren. Bei Thrombosen mit transientem Auslöser ging die Übersterblichkeit danach allmählich zurück, bei idiopathischen Thrombosen blieb die Mortalität bis zu acht Jahre lang mehr als verdoppelt. Im Schnitt wurde die Lebenserwartung durch eine Venenthrombose ohne Krebserkrankung um fünf Jahre gesenkt, von 81 auf 76 Jahre bei Männern und von 84 auf 79 bei Frauen.

Die führende Todesursache bei den Patienten mit Venenthrombosen waren Malignome (65\%), gefolgt von HerzKreislauf- und Atemwegserkrankungen (13\% und 6\%). Allein mit Komorbiditäten ließ sich die erhöhte Sterblichkeit der Thrombosepatienten jedoch nicht erklären. Auch Patienten mit idiopathischer Thrombose und ohne Begleiterkrankungen hatten eine schlechtere Prognose quoad vitam. Die Studienautoren empfehlen deswegen, Patienten mit einer ersten Venenthrombose oder Lungenembolie langfristig zu überwachen.

$\mathrm{BS}$.

- Quelle: Flinterman LE et al. Long-Term Survival in a Large Cohort of Patients with Venous Thrombosis: Incidence and Predictors. PLoS Med 2011; 9(1): e1001155. doi:10.1371/journal. pmed.1001155 